Pacific Journal of Mathematics

GEOMETRIC PROPERTIES OF SOBOLEV MAPPINGS 


\title{
GEOMETRIC PROPERTIES OF SOBOLEV MAPPINGS
}

\author{
RONALD GARIEPY
}

If $f$ is a mapping from an open $k$-cube in $R^{k}$ into $R^{n}$, $2 \leqq k \leqq n$, whose coordinate functions belong to appropriate Sobolev spaces, then the area of $f$ is the integral with respect to $k$ dimensional Hausdorff measure over $R^{n}$ of a nonnegative integer valued multiplicity function.

1. Introduction. If $f: Q \rightarrow R^{n}, Q$ an open $k$-cube in $R^{k}$, $2 \leqq k \leqq n$, is a mapping whose coordinate functions belong to appropriate Sobolev classes, it was shown in [6] that $f$ is $k-1$ continuous and that the area of $f$, as defined in [5], is equal to the classical Jacobian integral. The purpose of this paper is to investigate, using the theory of currents as in [2], the geometric-measure theoretic properties of such a surface and to show that the area is equal to the integral with respect to $k$ dimensional Hausdorff measure in $R^{n}$ of an integer valued multiplicity function.

2. Suppose $k$ and $n$ are integers with $2 \leqq k \leqq n$. Let

$$
Q=R^{k} \cap\left\{x: 0<x_{i}<1 \text { for } 1 \leqq i \leqq k\right\}
$$

and let $\Lambda(k, n)$ denote the set of all $k$-tuples $\lambda=\left(\lambda_{1}, \cdots, \lambda_{k}\right)$ of integers such that $1 \leqq \lambda_{1}<\cdots<\lambda_{k} \leqq n$. Suppose $f: Q \rightarrow R^{n}, f=$ $\left(f^{1}, \cdots, f^{n}\right), \quad f^{i} \in W_{p_{i}}^{1}(Q), \quad p_{i}>k-1, \quad$ and $\quad \sum_{j=1}^{k} 1 / p_{\lambda_{j}} \leqq 1$ whenever $\lambda \in \Lambda(k, n)$. Here $W_{p}^{1}(Q)$ denotes those functions in $L^{p}(Q)$ whose distribution partial derivatives are functions in $L^{p}(Q)$.

Let $e_{1}, \cdots, e_{n}$ be the usual basis for $R^{n}$ and let

$$
e_{\lambda}=e_{\lambda_{1}} \wedge \cdots \wedge e_{\lambda_{k}},
$$

$\lambda \in \Lambda(k, n)$, denote the corresponding basis for the space of $k$-vectors in $R^{n}$. For $\lambda \in \Lambda(k, n)$ let $p^{\lambda}$ denote the orthogonal projection of $R^{n}$ onto $R^{k}$ defined by letting

$$
p^{\lambda}(y)=\left(y_{\lambda_{1}}, \cdots, y_{\lambda_{k}}\right) \text { for } y=\left(y_{1}, \cdots, y_{n}\right) \in R^{n} \text {. }
$$

For almost every (in the sense of $k$ dimensional Lebesgue measure $\left.\mathscr{L}_{k}\right) x \in Q$, let $J f(x)=\sum_{\lambda \in \Lambda(k, n)} J f^{\lambda}(x) e_{\lambda}$ where $J f^{\lambda}$ denotes the determinant of the matrix of distribution partial derivatives of $f^{\lambda}=p^{\lambda} \circ f$. In [6] it was shown that the area of $f$, as defined in [5] is equal to $\int_{Q}|J f(x)| d x$ where $|J f(x)|$ is the Euclidean norm of the $k$-vector $J f(x)$. 
Define a current valued measure $T$ over $Q$ by letting

$$
T(B)(\phi)=\int_{B} \phi(f(x)) \cdot J f(x) d x
$$

whenever $B$ is an $\mathscr{L}_{k}$ measurable subset of $Q$ and $\phi$ is an infinitely differentiable $k$-form on $R^{n}$ with compact support. Let $\sigma$ denote the finite measure defined over $R^{n}$ by letting

$$
\sigma(Y)=\int_{f^{-1}(Y)}|J f(x)| d x
$$

whenever $Y$ is a Borel subset of $R^{n}$.

It will be shown in part 3 that $T(B)$ is a locally rectifiable current whenever $B$ is an $\mathscr{L}_{k}$ measurable subset of $Q$ and this fact will be used to define a nonnegative integer valued function $N$ on $R^{n}$ which describes the multiplicity with which $f$ assumes its values. The main results of the paper are summarized here.

THEOREM. Let $H_{n}^{k}$ denote $k$ dimensional Hausdorff measure in $R^{n}$ and let $\alpha(k)$ denote the $\mathscr{L}_{k}$ measure of the unit ball in $R^{k}$.

1. For $H_{n}^{k}$ almost every $y \in R^{n}$

$$
N(y)=\lim _{r \rightarrow 0+} \frac{\sigma(B(y, r))}{\alpha(k) r^{k}} .
$$

Here $B(y, r)$ denotes the open ball of radius $r$ around $y$.

2.

$$
\int_{R^{n}} N(y) d H_{n}^{k} y=\int_{Q}|J f(x)| d x .
$$

3. There exists a countable family $F$ of $k$ dimensional $C^{1}$ submanifolds of $R^{n}$ such that for $\sigma$ almost every $y \in R^{n}$ there is an $M \in F$ with $y \in M$,

$$
\lim _{r \rightarrow 0+} \frac{\sigma(B(y, r)-M)}{\alpha(k) r^{k}}=0
$$

and

$$
\lim _{r \rightarrow 0+} \frac{\sigma(B(y, r) \cap M)}{\alpha(k) r^{k}}=N(y)
$$

3. Definition of the function $N$ and proof of the theorem. We follow a plan analogous to that of [2: 2.1]. For $1 \leqq i \leqq k$ and $r \in I=\{s: 0<s<1\}$ let $P_{i}(r)=Q \cap\left\{x: x_{i}=r\right\}$. Let $\left\{f_{j}\right\}$ be a sequence of mollifiers of $f$ as in [6] and let $\bar{f}$ denote the pointwise limit of $\left\{f_{j}\right\}$ wherever it exists. Then $\bar{f}$ is a representative of $f$ and according to [6], [7: Chap. 3], and [8: part 3] there exists a collection 
$P$ of the sets $P_{i}(r)$ such that for each $i, P_{i}(r) \in P$ for almost all (in the sense of 1 dimensional Lebesgue measure) $r \in I$ and if $q$ is any $k$-cube in $Q$ whose $k-1$ faces all lie in elements of $P$ then

(1) $f_{j} \mid$ Bdry $q$ converges uniformly to $\bar{f} \mid$ Bdry $q$,

(2) $H_{n}^{k}(\bar{f}(\operatorname{Bdry} q))=0$

(3) $\quad L_{k-1}(\bar{f} \mid \operatorname{Bdry} q)=\underline{\lim }_{j \rightarrow \infty} L_{k-1}\left(f_{j} \mid \operatorname{Bdry} q\right)$, where $L_{k-1}$ denotes $k-1$ dimensional Lebesgue area.

Henceforth we will denote by $f$ the pointwise limit of mollifiers $\left\{f_{j}\right\}$ as described above. A $k$-cube $q \subset Q$ whose $k-1$ faces all lie in elements of $P$ will be called "special".

For the notation concerning currents we refer to [3].

Lemma 1. If $f$ is bounded then $T(B)$ is a rectifiable current whenever $B$ is an $\mathscr{L}_{k}$ measurable subset of $Q$.

Proof. If $q \subset Q$ is a special $k$-cube then

$$
\lim _{j \rightarrow \infty} \int_{q}\left|J f_{j}(x)-J f(x)\right| d x=0
$$

and hence the sequence $\left\{f_{j \neq}(q)\right\}$ of currents converges weakly to $T(q)$. The supports of the $f_{j \ddagger}(q)$ and $T(q)$ are all contained in a fixed compact set,

$$
M\left(f_{j \sharp}(q)\right) \leqq \int_{q}\left|J f_{j}(x)\right| d x,
$$

and

$$
M\left(\partial f_{j \sharp}(q)\right) \leqq L_{k-1}\left(f_{j} \mid \operatorname{Bdry} q\right)
$$

where $M$ denotes mass in the space of currents. Thus, by [4: 8.14, 8.13], $T(q)$ is an integral current whenever $q$ is special.

Since it is clear that

$$
M(T(A)) \leqq \int_{A}|J f(x)| d x
$$

whenever $A$ is an $\mathscr{L}_{k}$ measurable subset of $Q$, the lemma follows.

Let $\|T\|$ denote the finite measure over $Q$ defined by letting \| $T \|(A)$ denote the supremum of the numbers $\sum_{j=1}^{\infty} M\left(T\left(B_{j}\right)\right)$ taken over all countable disjoint collections of $\mathscr{L}_{k}$ measurable subsets $B_{j} \subset A$ whenever $A$ is an $\mathscr{L}_{k}$ measurable subset of $Q$. Clearly

$$
\|T\|(A) \leqq \int_{A}|J f(x)| d x
$$

whenever $A$ is an $\mathscr{L}_{k}$ measurable subset of $Q$.

For $\mathscr{L}_{k}$ almost every $x \in Q$ there is a $k$-covector $\omega$ in $R^{n}$ with 
$|\omega|=1$ such that $\omega \cdot J f(x)=|J f(x)|$ and

$$
\varlimsup_{r \rightarrow 0+} \frac{\|T\|(B(x, r))}{\alpha(k) r^{k}} \geqq \lim _{r \rightarrow 0+} \frac{T(B(x, r))(\omega)}{\alpha(k) r^{k}}=|J f(x)| .
$$

It follows that $\|T\|(A)=\int_{A}|J f(x)| d x$ whenever $A$ is an $\mathscr{L}_{k}$ measurable subset of $Q$.

For each positive integer $N$ let $f_{N}=\left(f_{N}^{1}, \cdots, f_{N}^{n}\right)$ where

$$
f_{N}^{i}(x)= \begin{cases}N & \text { if } f^{i}(x) \geqq N \\ f^{i}(x) & \text { if }\left|f^{i}(x)\right|<N \\ -N & \text { if } f^{i}(x) \leqq-N .\end{cases}
$$

Then $f_{N}$ is bounded and $f_{N}^{i} \in W_{p_{i}}^{1}(Q)$ for $1 \leqq i \leqq n$. For any measurable set $B \subset Q$ let

$$
T_{N}(B)(\phi)=\int_{B} \dot{\phi}\left(f_{N}(x)\right) \cdot J f_{N}(x) d x
$$

whenever $\dot{\phi}$ is an infinitely differentiable $k$-form on $R^{n}$. Note that, if $Y$ is a bounded Borel set in $R^{n}$, then, for sufficiently large $N$, $T_{N}(B)\left\llcorner Y=T(B)\left\llcorner Y\right.\right.$ whenever $B$ is an $\mathscr{L}_{k}$ measurable subset of $Q$. Consequently, if $Y$ is a bounded open subset of $R^{n}$ then $T(B)\llcorner Y$ is rectifiable whenever $B$ is a measurable subset of $Q$.

Analogous to [2: 2.1 part 3] we have

Lemma 2. There exists a countable collection $F$ of $k$ dimensional $C^{1}$ submanifolds of $R^{n}$ such that $\sigma\left(R^{n}-U F\right)=0$.

Proof. Suppose $r$ and $\varepsilon$ are positive real numbers and let

$$
B(0, r)=R^{n} \cap\{y:|y|<r\} .
$$

Let $\left\{A_{1}, \cdots, A_{m}\right\}$ denote a finite collection of disjoint measurable subsets of $f^{-1}(B(0, r))$ such that $\mathscr{L}_{k}\left(f^{-1}(B(0, r))-\bigcup_{j=1}^{m} A_{j}\right)=0$ and $\sigma(B(0, r))-\varepsilon<\sum_{j=1}^{m} M\left(T\left(A_{j}\right)\right)$. Note that $T\left(A_{j}\right)=T\left(A_{j}\right)\llcorner B(0, r)$ is rectifiable for $j=1, \cdots, m$. Thus, by [4:8.16], there exists for each $j$ a countable collection $G_{j}$ of $k$-dimensional $C^{1}$ submanifolds of $R^{n}$ such that $\left\|T\left(A_{j}\right)\right\|\left(R^{n}-\bigcup G_{j}\right)=0$. Letting $G=\bigcup_{j=1}^{m} G_{j}$, we have

$$
\begin{aligned}
\varepsilon & \geqq \sigma(B(0, r))-\sum_{j=1}^{m} M\left(T\left(A_{j}\right)\right)=\sum_{j=1}^{m}\left(\|T\|\left(A_{j}\right)-M\left(T\left(A_{j}\right)\right)\right) \\
& \geqq \sum_{j=1}^{m}\|T\|\left(A_{j} \cap f^{-1}\left(R^{n}-\bigcup G_{j}\right)\right) \\
& \geqq \sum_{j=1}^{m}\|T\|\left(A_{j} \cap f^{-1}\left(R^{n}-\bigcup G\right)\right)=\sigma(B(0, r)-\bigcup G)
\end{aligned}
$$


and the lemma follows.

If $\mu$ is a measure over $R^{n}, y \in R^{n}$, and $A \subset R^{n}$ we let

$$
\theta^{k}(\mu, A, y)=\lim _{r \rightarrow 0+} \frac{\mu(A \cap B(y, r))}{\alpha(k) r^{k}}
$$

whenever the limit exists. In case $A=R^{n}$ we write $\theta^{k}(\mu, y)$.

Recall that, if $S$ is a $k$ dimensional rectifiable current in $R^{n}$ and $Y$ is a Borel set in $R^{n}$ with $H_{n}^{k}(Y)=0$, the $S L Y=0$. Thus $\sigma$ is absolutely continuous with respect to $H_{n}^{k}$. This fact together with Lemma 2 and the finiteness of $\sigma$ allow us to conclude using [1: 3.1, 3.2] that:

1. $\theta^{k}(\sigma, y)$ exists for $H_{n}^{k}$ almost every $y \in R^{n}$.

2. For $\sigma$ almost every $y \in R^{n}$ there exists an $M \in F$ such that $y \in M, \theta^{k}(\sigma, y)<\infty$, and $\theta^{k}\left(\sigma, R^{n}-M, y\right)=0$.

3. $\int_{R^{n}} \theta^{k}(\sigma, y) d H_{n}^{k} y \leqq \sigma\left(R^{n}\right)$.

A proof of the following statement concerning rectifiable currents can be found in [2:2.1 part 7]: If $S$ is a rectifiable $k$ dimensional current in $R^{n}, M$ is a $k$ dimensional $C^{1}$ submanifold of $R^{n}$,

$$
y \in M-\operatorname{spt} \partial S,
$$

$\theta^{k}(\|S\|, y)<\infty, \theta^{k}\left(\|S\|, R^{n}-M, y\right)=0, \quad$ and $P$ is an oriented $k$ plane tangent to $M$ at $y$, then there exists a unique integer $m$ such that

$$
\lim _{r \rightarrow 0+} \frac{1}{\alpha(k) r^{k}} F[S\llcorner B(y, r)-m(P \cap B(y, r))]=0
$$

where $F$ denotes the flat norm [4: 3.2].

Now suppose $q$ is a special $k$-cube in $Q$ and $y \in R^{n}$. If there is an $M \in F$ with $y \in M-f(\operatorname{Bdry} q), \theta^{k}(\sigma, y)<\infty$, and

$$
\theta^{k}\left(\sigma, R^{n}-M, y\right)=0,
$$

let $P$ denote an oriented $k$-plane tangent to $M$ at $y$, let $m(q, y)$ denote the integer such that

$$
\lim _{r \rightarrow 0+} \frac{1}{\alpha(k) r^{k}} F[T(q)\llcorner B(y, r)-m(q, y)(P \cap B(y, r))]=0
$$

and set $\alpha(q, y)=m(q, y) \zeta(y)$ where $\zeta(y)$ is the simple unit $k$-vector orienting $P$. Otherwise set $\alpha(q, y)=0$.

Then, for $H_{n}^{k}$ almost every $y \in R^{n}$,

$$
\theta^{k}\left(T(q)\llcorner\dot{\phi}, y)=\lim _{r \rightarrow 0} \frac{[T(q)\llcorner B(y, r)](\phi)}{\alpha(k) r^{k}}=\phi(y) \cdot \alpha(q, y)\right.
$$


whenever $\phi$ is an infinitely differentiable $k$-form in $R^{n}$. Consequently $T(q)(\phi)=\int_{R^{n}} \phi(y) \cdot \alpha(q, y) d H_{n}^{k} y$ whenever $\phi$ is an infinitely differentiable $k$-form and hence

$$
M(T(q)) \leqq \int_{R^{n}}|\alpha(q, y)| d H_{n}^{k} y
$$

whenever $q$ is a special $k$-cube.

For $y \in R^{n}$ let $N(y)$ denote the supremum of the numbers $\sum_{q \in G}|\alpha(q, y)|$ taken over all finite collections $G$ of nonoverlapping special $k$-cubes in $Q$.

Suppose $N(y) \neq 0$ and $G$ is a finite collection of nonoverlapping special $k$-cubes such that $\alpha(q, y) \neq 0$ for $q \in G$. Let $\omega$ denote a $k$ covector with $|\omega|=1$ and $\omega \cdot \zeta(y)=1$. Then

$$
\begin{aligned}
\sum_{q \in G}|\alpha(q, y)| & =\sum_{q \in G} \mid \theta^{k}(T(q)\llcorner\omega, y) \mid \\
& =\lim _{r \rightarrow 0} \sum_{q \in G} \frac{\mid[T(q)\llcorner B(y, r)](\omega) \mid}{\alpha(k) r^{k}} \\
& \leqq \theta^{k}(\sigma, y) .
\end{aligned}
$$

Thus $N(y) \leqq \theta^{k}(\sigma, y)$ for $H_{n}^{k}$ almost every $y \in R^{n}$.

On the other hand, if $G$ is any finite collection of nonoverlapping special $k$-cubes,

$$
\sum_{q \in G} M(T(q)) \leqq \int_{R^{n}} \sum_{q \in G}|\alpha(q, y)| d H_{n}^{k} y .
$$

The supremum of the numbers $\sum_{q \in G} M(T(q))$ taken over all finite collections $G$ of nonoverlapping special $k$-cubes is readily seen to be $\sigma\left(R^{n}\right)$ and hence

$$
\sigma\left(R^{n}\right) \leqq \int_{R^{n}} N(y) d H_{n}^{k} y \leqq \int_{R^{n}} \theta^{k}(\sigma, y) d H_{n}^{k} y \leqq \sigma\left(R^{n}\right) .
$$

Thus $N(y)=\theta^{k}(\sigma, y) H_{n}^{k}$ almost everywhere and

$$
\int_{R^{n}} N(y) d H_{n}^{k} y=\int_{Q}|J f(x)| d x
$$

\section{REFERENCES}

1. H. Federer, The $(\phi, k)$ rectifiable subsets of $n$ space, Trans. Amer. Math. Soc., 62 (1947), 441-466.

2. —, Currents and area, Trans. Amer. Math. Soc., 98 (1961), 204-233.

3. —_ Geometric Measure Theory, Springer-Verlag, New York, 1969.

4. H. Federer and W. Fleming, Normal and integral currents, Ann. of Math., 72 (1960), 458-520.

5. C. Goffman and F. C. Liu, Discontinuous mappings and surface area, Proc. London Math. Soc., 20 (1970), 237-248. 
6. C. Goffman and W. P. Ziemer, Higher dimensional mappings for which the area formula holds, Ann. of Math., 92 (1970), 482-488.

7. C. B. Morrey Jr., Multiple Integrals in the Calculus of Variations, Springer-Verlag, New York, 1966.

8. Y. G. Reshetnyak, Space mappings with bounded distortion, Siberian Math. J., 8 (1967), 446-487.

Received March 7, 1972.

UNIVERSITY OF KENTUCKY 



\section{PACIFIC JOURNAL OF MATHEMATICS}

\section{EDITORS}

D. Gilbarg AND J. MILGRAM

Stanford University

Stanford, California 94305

\section{R. A. Beaumont}

University of Washington

Seattle, Washington 98105

\section{J. DUGUNDJI*}

Department of Mathematics University of Southern California Los Angeles, California 90007

RICHARD ARENS

University of California Los Angeles, California 90024

\section{ASSOCIATE EDITORS}
E. F. BECKENBACH
B. H. NEUMANN
F. WOLF
K. YoshidA

\section{SUPPORTING INSTITUTIONS}

\author{
UNIVERSITY OF BRITISH COLUMBIA \\ CALIFORNIA INSTITUTE OF TECHNOLOGY \\ UNIVERSITY OF CALIFORNIA \\ MONTANA STATE UNIVERSITY \\ UNIVERSITY OF NEVADA \\ NEW MEXICO STATE UNIVERSITY \\ OREGON STATE UNIVERSITY \\ UNIVERSITY OF OREGON \\ OSAKA UNIVERSITY
}

\author{
UNIVERSITY OF SOUTHERN CALIFORNIA \\ STANFORD UNIVERSITY \\ UNIVERSITY OF TOKYO \\ UNIVERSITY OF UTAH \\ WASHINGTON STATE UNIVERSITY \\ UNIVERSITY OF WASHINGTON \\ AMERICAN MATHEMATICAL SOCIETY \\ NAVAL WEAPONS CENTER
}

The Supporting Institutions listed above contribute to the cost of publication of this Journal, but they are not owners or publishers and have no responsibility for its content or policies.

Mathematical papers intended for publication in the Pacific Journal of Mathematics should be in typed form or offset-reproduced, (not dittoed), double spaced with large margins. Underline Greek letters in red, German in green, and script in blue. The first paragraph or two must be capable of being used separately as a synopsis of the entire paper. Items of the bibliography should not be cited there unless absolutely necessary, in which case they must be identified by author and Journal, rather than by item number. Manuscripts, in duplicate if possible, may be sent to any one of the four editors. Please classify according to the scheme of Math. Rev. Index to Vol. 39. All other communications to the editors should be addressed to the managing editor, Richard Arens, University of California, Los Angeles, California, 90024.

50 reprints are provided free for each article; additional copies may be obtained at cost in multiples of 50 .

The Pacific Journal of Mathematics is issued monthly as of January 1966. Regular subscription rate: $\$ 48.00$ a year (6 Vols., 12 issues). Special rate: $\$ 24.00$ a year to individual members of supporting institutions.

Subscriptions, orders for back numbers, and changes of address should be sent to Pacific Journal of Mathematics, 103 Highland Boulevard, Berkeley, California, 94708.

PUBLISHED BY PACIFIC JOURNAL OF MATHEMATICS, A NON-PROFIT CORPORATION

Printed at Kokusai Bunken Insatsusha (International Academic Printing Co., Ltd.), 270, 3-chome Totsuka-cho, Shinjuku-ku, Tokyo 160, Japan.

* C. DePrima will replace J. Dugundji until August 1974.

Copyright (C) 1973 by

Pacific Journal of Mathematics

All Rights Reserved 


\section{Pacific Journal of Mathematics}

\section{Vol. 47, No. $2 \quad$ February, 1973}

David Parham Bellamy, Composants of Hausdorff indecomposable continua; a mapping approach ........................ 303

Colin Bennett, A Hausdorff-Young theorem for rearrangement-invariant spaces ...........................................

Roger Daniel Bleier and Paul F. Conrad, The lattice of closed ideals and $a^{*}$-extensions of an abelian l-group ...

Ronald Elroy Bruck, Jr., Nonexpansive projections on subsets of Banach

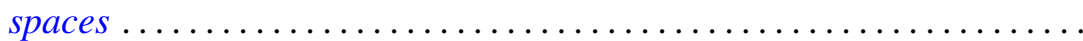

Robert C. Busby, Centralizers of twisted group algebras ............. 357

M. J. Canfell, Dimension theory in zero-set spaces ................ 393

John Dauns, One sided prime ideals ........................ 401

Charles F. Dunkl, Structure hypergroups for measure algebras . . . . . . . . . 413

Ronald Francis Gariepy, Geometric properties of Sobolev mappings ...... 427

Ralph Allen Gellar and Lavon Barry Page, A new look at some familiar spaces of intertwining operators ...........................

Dennis Michael Girard, The behavior of the norm of an automorphism of the

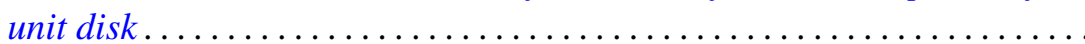

George Rudolph Gordh, Jr., Terminal subcontinua of hereditarily

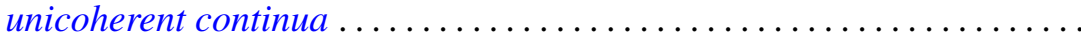

Joe Alston Guthrie, Mapping spaces and cs-networks. .

Neil Hindman, The product of $F$-spaces with $P$-spaces . 473

M. A. Labbé and John Wolfe, Isomorphic classes of the spaces $C_{\sigma}(S)$

Ernest A. Michael, On k-spaces, $k_{R}$-spaces and $k(X) \ldots$

Donald Steven Passman, Primitive group rings .

C. P. L. Rhodes, A note on primary decompositions of a pseudovaluation ...

Muril Lynn Robertson, A class of generalized functional differential equations

Ruth Silverman, Decomposition of plane convex sets. $I$.

Ernest Lester Stitzinger, On saturated formations of solvable Lie algebras................................

B. Andreas Troesch, Sloshing frequencies in a half-space by Kelvin inversion ...

L. E. Ward, Fixed point sets .

Michael John Westwater, Hilbert transforms, and a problem in scattering

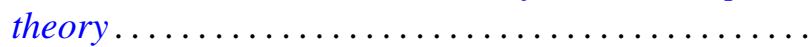

Misha Zafran, On the spectra of multipliers... 